\title{
Kernos
}

Revue internationale et pluridisciplinaire de religion grecque antique

5 | 1992

Varia

\section{Prévenir ou guérir ? Musique et états orgiastiques chez Platon}

\section{Evanghelos A. Moutsopoulos}

\section{OpenEdition \\ Journals}

\section{Édition électronique}

URL : http://journals.openedition.org/kernos/1056

DOI : 10.4000/kernos. 1056

ISSN : 2034-7871

\section{Éditeur}

Centre international d'étude de la religion grecque antique

\section{Édition imprimée}

Date de publication : 1 janvier 1992

ISSN : 0776-3824

\section{Référence électronique}

Evanghelos A. Moutsopoulos, "Prévenir ou guérir ? Musique et états orgiastiques chez Platon », Kernos [En ligne], 5 | 1992, mis en ligne le 19 avril 2011, consulté le 21 avril 2019. URL : http:// journals.openedition.org/kernos/1056; DOI : 10.4000/kernos.1056 


\section{PRÉVENIR OU GUÉRIR ? MUSIQUE E'T ÉTATS ORGIASTIQUES CHEZ PLATON}

L'on sait que dans toutes les sociétés archaïques ${ }^{1}$, la musique est fonctionnellement omniprésente à tous les niveaux culturels et sociaux. Il ne saurait en être autrement dans les sociétés grecques classiques qui, non seulement, dérivaient des sociétés grecques archaïques à proprement parler, mais se trouvaient aussi en contact ininterrompu avec les sociétés "barbares" qui peuplaient la périphérie géographique de l'hellénisme ${ }^{2}$, et dont l'apport culturel à l'Hellade ne fut pas négligeable. Qu'ils l'aient regretté ou non, les philosophes, Platon en tête, furent obligés de la reconnaitre ${ }^{3}$. Certes, dans les manifestations de type apollinien, pour reprendre la distinction nietzschéenne, l'influence "barbare" fut de bonne heure plus ou moins limitée aux domaines de l'esthétique et de quelques techniques artistiques; mais des manifestations de type dionysiaque, il fut impossible de l'exclure, tant les pratiques grecques pouvaient se confondre avec des pratiques extrahelléniques. D'ailleurs, Dionysos lui-même ne fut-il pas un dieu "d'importation", de même que les cultes "bachiques» 4 ? Or tous ces cultes, comme les pratiques qui les accompagnaient, reposaient sur la possibilité de susciter des états orgiastiques où la musique jouait un rôle prépondérant ${ }^{5}$. Platon en fut pleinement conscient.

Pour la pensée platonicienne, musique et magie sont des activités étroitement liées: la musique exercerait d'elle-même un tel pouvoir magique ${ }^{6}$ qu'elle était quasiment prédestinée à s'associer à la magie. Platon considère les chants comme des incantations. C'est à maintes

1 Sur la préférence accordée à ce terme par rapport aux termes «inférieures", "pré-logiques», "primitives» (L. Lévy-Bruhl), "sauvages» (Cl. Lévi-Strauss), etc., cf. J. CAZENEUVE, La mentalité archaïque, Paris, 1961, p. 17 sq.

2 E. Moursopoulos, Musique grecque ou «barbare» (Eurip., Iph. Taur., 179 184)?, in Eirene, 21 (1984), p. 25-31; Diotima, 12 (1984), p. 158-163.

3 ID., La musique dans l'ouvre de Platon, Paris, 19892, p. 11 sq.

4 H. JEANMAIRE, Le Satyre et la Ménade. Remarques sur quelques textes relatifs aux danses "orgiaques", in Mélanges Charles Picard, tome II, Paris, 1949, p. 463-473.

5 E. Moutsopoulos, La philosophie de la musique dans la dramaturgie grecque. Formation et structure, Athènes, 1975, p. 18; 100 sq.; 97 et la n. 4. 
reprises qu'il confirme cette identification ${ }^{7}$, et c'est pour cette même raison qu'il recommande à tous les citoyens de pratiquer des incantations pour obtenir l'harmonie de leurs âmes. L'étymologie du terme d'épodè confirme qu'à l'origine les incantations étaient principalement des chants issus de formules magiques ou même sacrées, et adressés à quelque puissance surnaturelle afin de la contraindre à être favorable ou, au contraire, d'en prévenir l'action pernicieuse ${ }^{8}$. Par contre, épodos serait simplement le refrain qui scande la suite de vers récités. Or cette fonction littéraire ne masque-telle pas précisément une fonction magique originelle dont témoignent d'innombrables pratiques religieuses relevées par les ethnographes? Dans le Charmide, Platon considère les incantations comme des moyens de guérison autant que comme discours philosophiques ${ }^{9}$. Il rejoint par là sa conception, exposée dans le Phédon, que la philosophie est la musique suprême ${ }^{10}$.

L'emploi d'incantations dans un but médical ne saurait être négligé, notamment en tant que technique de guérison des blessures ${ }^{11}$. Zalmoxis fut surtout un guérisseur qui eut recours à des incantations ${ }^{12}$. Orphée fut un chanteur parce qu'il fut «un enchanteur» qui savait charmer non seulement les animaux, mais aussi les guerriers thraces ${ }^{13}$. Il fut, avec Musée, le premier à avoir connu les herbes médicinales ${ }^{14}$. Purificateur, musicien, danseur tout à la fois, il fut

7 Platon, Lois, II, 659e; 664b; 665e; 666c; 670e; 927a; cf. III, 677d où les incantations sont considérées comme les vestiges des techniques musicales mises au point par des civilisations disparues.

8 De même, carmen signifie en latin le chant autant que le charme. Cf. E. Moutsopoulos, Musique et musicalité dans les "Oracles chaldaïques", in Kernos, 3 (1990), p. 281-293, notamment p. 288; J. COMBARIEU, La musique et la magie, Paris, 1909, p. 22 : «on les [formules magiques] a d'abord chantées; puis... récitées; enfin, écrites»; P. Boyance, Le culte des Muses chez les philosophes grecs, Paris, 1972, p. 41 : «souvent, à défaut de... chant..., on a cru nécessaire une certaine intonation, certains caractères de débit».

9 Platon, Charmide, 156d; 175e.

10 ID., Phédon, 61a.

11 P.-M. ScHuHL, Essai sur la formation de la pensée grecque, Paris, 1933, p. 71.

12 Platon, Charmide, 156d.

13 P.-M. SchuHL, Les premières étapes de la philosophie biologique, in Revue d'Histoire des Sciences, 4 (1950), p. 197-221, notamment p. 208.

14 Theophr., Hist. plantes, IX, 19, 2; Pline, Hist. nat., XXI, 20 (145); XXV, 2 (12). Cf. Platon, Lois, VI, 782e, qui, par ailleurs, s'insurge contre les contrefaçons de l'orphisme pratiquées de son temps par divers guérisseurs ambulants : Rép., II, 364b-c; Lois, X, 908d; 909a-d. 
suivi par les Pythagoriciens qui instituèrent «une véritable pharmacopée musicale» connue par Platon, à travers les Pythagoriciens italiens ${ }^{15}$ et reposant sur l'association entre musique et magie. Au demeurant, Platon ne transpose-t-il pas les incantations orphiques et pythagoriciennes «en mythes» 16 ? Ici, comme partout ailleurs quand il s'agit de magie (sympathique), une structure homologique est sousentendue comme intervenant simultanément à deux niveaux ${ }^{17}$. Remède contre la maladie, capable de restituer l'harmonie du fonctionnement dérangé de l'organisme, l'incantation, partant l'harmonie musicale, restitue la prudence au niveau de l'âme ${ }^{18}$. Platon lui réserve une place curative et culturelle exceptionnelle dans sa cité ${ }^{19}$. Il l'élève ainsi à un rang spirituel à l'encontre des pratiques magiques en usage de son temps, censées exercer un véritable pouvoir sur les forces naturelles.

Le terme même de télétai aurait à l'origine désigné des formules chantées, transmises aux adeptes lors des mystères. Plus les incantations seraient puissantes et plus elles établiraient un lien étroit entre l'homme et la divinité invoquée. Celles d'Orphée l'auraient été infinement, puisqu'elles auraient suffi pour le conduire aux Enfers. Suaves, comme celles de Thamyras, elles pourraient servir de modèles à d'autres compositions; mais pour être exécutées, celles-ci devraient être approuvées au préalable ${ }^{20}$. On retiendra la double fonction, invocatrice et apaisante des incantations. Or, longtemps avant Platon, l'élaboration de la conception orphique de la magie musicale par les Pythagoriciens aurait conduit les recherches de ces derniers dans une nouvelle direction, par la création "d'un vocabulaire à la fois technique et mystique ${ }^{21}$ » qui devait aboutir à "une synthèse des croyances disparates sur la magie du nombre, de l'astronomie et de la musique ${ }^{22}$ ".

15 P.-M. ScHuHL, Essai..., op. cit.; E. MouTsopoulos, La catharsis tragique chez Aristote : une énigme?, in Diotima, 2 (1974), p. 248-250.

16 ID., Etudes sur la fabulation platonicienne, Paris, 1947, p. 9; P. BoYANCE, Le culte des Muses..., op. cit., p. 145; E. Moutsopoulos, La musique..., op. cit., p. 14 et la n. 12.

17 E. Moutsopoulos, Structures intimes et plaisir esthétique. Kairos, la mise et l'enjeu, Paris, Vrin, 1991, chap. 33.

18 Platon, Timée, 80b.

19 ID., Phédon, 85a sq.; 77e; 114d; Rép., II, 376e; 377a-d; III, 414c; 415c; Lois, 887d; 903a; 927a.

20 ID., Lois, VIII, 829e.

21 E. Moutsopoulos, La musique..., op. cit., p. 16.

22 Ibid. 
Platon se rend à cette évidence sans se douter que, déjà au dire d'Hérodote ${ }^{23}$, il y avait eu confusion au sujet de rites pythagoriciens, d'origine vraisemblablement égyptienne, qui passaient pour orphiques et dionysiaques ${ }^{24}$. Toutefois, la magie musicale opère dans deux sens complémentaires: elle facilite l'invocation de la divinité, mais elle provoque aussi l'inspiration poétique.

Inspiré par la Muse, le poète cesse d'être maître de son esprit ${ }^{25}$, il est rendu enthousiaste; il est en proie à un délire comparable à celui des Corybantes 26 en état de transe. Pris de transports bachiques, ils s'apparentent «aux Bacchantes... lorsqu'elles sont possédées ${ }^{27}$ ». De fait, «les possédés des Corybantes tombent en transe lorsque se fait entendre la mélodie propre à la divinité dont ils sont possédés ${ }^{28}$... Cet état de possession... se manifeste par une mimique et des propos dans lesquels il est difficile de voir autre chose que la manifestation même de l'être démoniaque qui les possède $\mathrm{e}^{29}$ ». Le poète est un intermédiaire entre la divinité inspiratrice et son propre groupe social auquel il transmet sa folie réductible à l'une des quatre catégories de la possession poétique, attribuée aux Muses, les autres étant les possessions mantique, érotique et télestique, respectivement attribuable à Apollon, à Aphrodite et Éros, et à Dionysos ${ }^{30}$. Traitée convenablement ${ }^{31}$, l'inspiration se solde en incantation contre l'injustice ${ }^{32}$. La conception que Platon, après Damor d'Oa, se fait des effets de la musique repose entièrement sur une vision mécaniste de l'homologie des mouvements des sons, de l'âme et du corps.

23 HDT., II, 81 (testim. 216 Kern).

24 P. BOYANCE, op. cit., p. 93-94; 99.

25 Platon, Ion, 536c; Euthyd., 277d-e; Phédon, 60e; Banquet, 215e; Phèdre, 228d; 234d; Lois, IV, 719c; VII, 790d-791e. Cf. I.M. LINFORTH, The Corybantic Rites in Plato, in CSCA, 13 (1946), p. 121-161.

26 Platon, Criton, 54d.

27 ID., Ion, 533e-534c; Banquet, 196d-197b.

28 ID., Criton, $54 \mathrm{~d}$.

29 H. Jeanmaire, Dionysos. Histoire du culte de Bacchus, Paris, 1951, p. 134. Cf. Plation, Phèdre, 244d-e.

30 Platon, Phèdre, 265a-b.

31 Ibid., 243a-b, où il est rapporté qu'ayant perdu la vue, Stésichore se serait luimême guéri de son mal en recourant à un remède musical de son invention : la palinodie.

32 ID., Ménex, 239b; Rép., I, 335c; III, 410a-b; 411c-d; Lois, VII, 817. Les phronimoi (Rép., I, 349e) et les emphronès (Timée, 80b) opposés aux aphronès rappellent les kompsoi opposés aux idiotai (Théet., 171d), privés eux de culture musicale. 
Selon cette conception, les sons se propageraient à travers l'air à une vitesse proportionnelle à leur hauteur. Cette vitesse serait sujette à un ralentissement également proportionnel dès que chaque son atteint et traverse le corps humain pour en inonder toutes les parties qui sont les sièges respectifs des parties de l'âme ${ }^{33}$. Les sons les plus graves et les plus lents, qui avaient atteint le corps longtemps après les sons les plus aigus et les plus rapides, les rattrapent pendant leur parcours dans le corps et restituent l'harmonie initiale dérangée à l'origine en raison du comportement diversifié des sons qui la composent, lors de leur passage dans l'air. Les effets de l'harmonie sur l'âme sont de deux sortes, selon la qualité du sujet qui les éprouve : l'insensé n'en ressent qu'un simple plaisir; le sage, lui, contemple, à travers l'harmonie sensible, l'harmonie idéale suprême ${ }^{34}$. Finalement, une structure artistique plairait à condition d'être homologue à une structure viscérale. Sonore ou orchestique, le mouvement musical entraînerait, par une synkinésis, une com-motion, un mouvement de l'âme, ainsi qu'Aristide Quintilien l'expliquera plus tard ${ }^{35}$ : homologie potentielle, sympathie actuelle.

Le passage du Criton qui mentionne l'enchantement que la parole des lois personnifiées exerce sur Socrate fait état des initiés aux mystères des Corybantes, acolytes de Cybèle, qui passaient pour avoir institué les mystères du même nom où l'initiation s'opérait moyennant les danses vertigineuses des prêtres autour de l'initié. Étourdi, celui-ci croyait entendre le son des flûtes du cortège divin. Mouvements sonores et mouvements corporels habilement combinés produiraient des situations à effet total, où l'équilibre corporel et mental ${ }^{36}$, déjà conseillé par les Pythagoriciens, est complètement rompu. Contre de tels mouvements nuisibles, un corps peut réagir par auto-immunisation ${ }^{37}$, comme aussi moyennant une éducation et un entraînement orchestiques convenables. À cet effet, on a recours à des mouvements réguliers. Ce sont eux, et non le repos, qui s'opposent aux mouvements déréglés causés

33 ID., Timée, 67a sq.; 80a-b. Cf. Y. BrEs, La psychologie de Platon, Paris, 1965, p. 87 sq., où l'apparition de la théorie platonicienne de la tripartition de l'âme est datée avec précision.

34 Cf. supra, et la n. 32.

35 Arist. Quint., De mus., p. 107 Meibom. Cf. E. Moutsopoulos, Sur la participation musicale chez Plotin, in Philosophia, 1 (1971), p. 379-389, notamment p. 384 et la n. 28.

36 Platon, Timée, 88b-c. Sur les effets physiologiques de la pénétration du chaud et du froid, du sec et de l'humide dans le corps humain, cf. Timée, 88d.

37 Ibid., 89a. 
soit par une défection de l'âme, soit par quelque possession corybantique $^{38}$. Le modèle typique du mouvement régulier, c'est l'aiorésis, le mouvement balancé du bateau qui navigue. Les mères qui veulent endormir leurs enfants qui s'agitent leur impriment un mouvement balancé des bras accompagné d'une berceuse. Les deux procédés combinés agissent à la manière d'une incantation et produisent l'apaisement souhaité.

Or il faut de la mesure en toutes choses : «il ne faut pas irriter les maladies par des remèdes quand elles n'offrent pas de grands dangers ${ }^{39}$ " et, partant, il faut que les mouvements rectificatifs soient "calculés de sorte qu'ils ne causent pas plus de mal que de bien 40 ". On se trouve en pleine problématique du métron, elle-même apparentée à celle du kairos. Toutefois, le recours au mouvement régulier, à plusieurs égards cathartique, est bénéfique aussi bien aux nourrissons encore privés de raison qu'aux possédés qui l'ont perdue. De Damon d'Oa, il est rapporté qu'il aurait guéri des jeunes gens de leur manie par un simple changement d'air musical ${ }^{41}$. Damon, comme les Pythagoriciens ${ }^{42}$, suivi par Platon, préconise en l'occurrence une méthode allopathique, alors qu'Aristote défendra une méthode homéopathique ayant, elle aussi, ses lettres de noblesse: «Le devin Mélampous... propagea le culte orgiaque et sut 'apaiser la furie' en la surexcitant par des danses sauvages pour mieux la maîtriser ensuite $^{43}$ ». Les mouvements violents imprimés au corps produisent une véritable frénésie. On rapprocherait de ces techniques celles qui, dans des cas analogues, sont encore en usage en Afrique. C'est dans des

38 Ibid., 88d-e; Lois, VII, 790c-791a; Philèbe, 34a. Il est surtout question des battements de cour violents qui traduisent la frayeur, état défectueux de l'âme s'il en est.

39 ID., Timée, 89a-b.

40 E. Moutsopoulos, La musique.., op. cit., p. 103.

41 F. LASSERre, Plutarque. De la musique, Olten-Lausanne, 1954, p. 63.

42 Tel Cleinias de Tarente contemporain de Platon, qui, s'il se trouvait en colère, obtenait la catharsis musicale en jouant de la lyre. Cf. P. BOYANCE, op. cit., p. 131.

43 ARISTOTe, Poétique, 1449b 27-28. Cf. P.-M. SCHUHL, Les premières étapes..., art. cit., p. 209; A. DELATTE, Les conceptions de l'enthousiasme chez les philosophes présocratiques, Paris, 1934, p. 70-71; P. BoYANCE, op. cit., p. 63-64. Platon, Phèdre, 223b; 244e-245a; Euthyd., 277d; Lois, II, 672b. Cf. I.M. LINFORTH, art. cit., p. 163-172. 
considérations semblables qu'on trouverait éventuellement l'explication du mépris affiché par Platon pour les danses orgiaques ${ }^{44}$.

Dans ces pratiques, il s'agissait de "substituer à l'état de possession latent et diffus... une possession explicitée... La danse de possession... est précisément cet état de transe réalisé sous le contrôle de guérisseurs..., chefs de... confréries et eux-mêmes grands possédés. Elle est considérée comme la danse de l'esprit possesseur... auquel le possédé, en donnant occasion à son maître de se manifester dans la mimique que comporte cette agitation violente, rend l'hommage qui le désarme et concilie ${ }^{45}$ ". Cette méthode homéopathique recourait donc à une musique et à une orchestique de plus en plus violentes, et qui réussissaient à guérir la peur par la peur. Le texte platonicien du livre VII des Lois et le texte aristotélicien de la Poétique, qui contient la fameuse définition de la tragédie, et que nous évoquons ici, mettent tous les deux en rapport les notions de peur et de purification. Purifiée, l'âme atteint son degré d'excellence qu'il lui faut maintenir en s'exerçant sans cesse. "Désormais toutes les fois qu'elle se manifestera par des mouvements corporels, ceux-ci seront réguliers, rythmés, harmonieux,

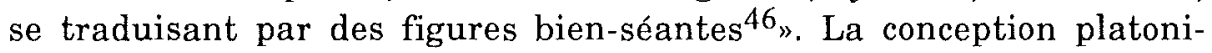
cienne du circuit des mouvements musicaux réfléchis s'insère dans la conception alcméonienne des cycles physiologiques, présente dans le Timée $e^{47}$.

Le sens du rythme, de l'harmonie et de l'ordre, on l'a vu, n'est pas inné; par contre, ce qui est inné, c'est la prédisposition à l'acquisition de la connaissance de toutes ces valeurs, et c'est cette prédisposition qui est le don d'Apollon et des Muses, selon le Finalisme que Platon affiche dans le Timée 48 . Il va même jusqu'à rapprocher les termes de choros (danse) et de chara (joie) ${ }^{49}$, dans le cadre d'une définition qui reflète directement les étymologies à la fois fantaisistes et savoureuses du Cratyle. Les dieux eux-mêmes deviennent de la sorte des chorèges, des donateurs et des protecteurs de la danse.

44 Ibid., p. 121 sq.

45 H. Jeanmaire, Le Satyre et la Ménade..., art. cit., p. 468.

46 E. Moutsopoulos, La musique..., op. cit., p. 110; H. JeAnMaIre, Dionysos..., op. cit., p. 316-321 (chap. Mimésis et catharsis).

47 Platon, Timée, 88c. Cf. Ch. Mugler, Alcméon et les cycles physiologiques de Platon, in REG, 71 (1958), p. 42-50.

48 Ibid., 37a; Lois, II, 653e; 664e. Apollon intervient ici en tant que guérisseur et Péan. Cf. Phèdre, 259c.

49 ID., Lois, II, 654a; VII, 816b sq.; Crat., 397c sq. 
D'inspiration et de caractère divins, musique et danse sont salutaires aux hommes. Chez ceux-ci, comme chez toutes les espèces animales, les jeunes sont incapables de tenir en repos leur corps et leur voix; ils crient et sautent en désordre. Or l'ordre du mouvement c'est le rythme. C'est à travers lui que les humains seuls sont capables de surmonter le désordre qui, initialement, règne en eux. Seuls les humains apprennent à maitriser leurs mouvements, comme leurs pensées ${ }^{50}$. Dans les sociétés grecques, la danse fut, elle aussi, comme d'ailleurs dans toutes les sociétés archaïques, une manifestation attachée à des rites ${ }^{51}$. "Lucien affirmera... qu'Orphée et Musée, qu'il appelle les plus parfaits danseurs de leur époque, avaient prescrit, en instituant les mystères, qu'ils eussent toujours lieu avec le rythme et la danse, et il ajoute qu'on ne saurait trouver d'initiation ancienne qui soit dépourvue d'un accompagnement orchestique ${ }^{52}$." Sa relation avec des pratiques religieuses n'est pas sans rapport avec ses vertus curatives ${ }^{53}$. Dans les Lois, Platon insiste longuement sur la vertu éducative de la danse: "celui qui n'a pas appris à danser, nous le considérons comme inculte, et pour qu'une personne soit jugée cultivée, il lui faudra avoir passablement dansé au préalable ${ }^{54}$.

C'est encore dans les Lois que Platon fixe les deux sortes de danses acceptables dans la Cité, l'une de caractère guerrier, la pyrrhique, l'autre de caractère religieux, l'emmélie qui, par son mouvement calme et ordonné, s'opposerait au caractère violent des danses bachiques. C'est deux types de danse sont qualifiées de politiques : elles promeuvent la vie de la Cité. Il en est de même des formes musicales qui, elles, devront être pures et conformes aux modèles classiques ${ }^{55}$ qui

50 Ibid, 653e; 664e.

51 Ibid., VII, 796c; 799a-b; 803e; 815d. Cf. B. LOWLER, The Maenads. A Contribution to the Study of Dance in Ancient Greece, in Memoirs of the American Academy in Rome, 6 (1927), p. 69-112.

52 L. SeChnN, La danse..., p. 39. Cf. Lucien, De la danse, 15.

53 E. Moutsopoulos, La musique..., op. cit., p. 107 et la n. 2, sur les danses qui accompagnaient les rites et l'initiation corybantiques.

54 PLATON, Lois, II, 654a; 672e; VII, 817a.

55 Il est à remarquer qu'à l'encontre des règles pour le choix de la bonne musique, dogmatiquement fixées dans la République, III, 397c sq., Platon, qui revient sur le problème à la fin de sa vie, dans les Lois, VII, 815a sq., y adopte une attitude qu'on qualifiera facilement de "pragmatisme", puisqu'il pose désormais comme critère de choix la longévité des formes : de toutes les formes sont pour lui préférables celles-là mêmes qui ont le mieux survécu aux ravages du temps. Cf. E. Moutsopoulos, Le modèle de l'œuvre d'art: idéalisme ou 
ne se prêtent nullement à des jugements d'ordre «théâtrocratique» 56 , et qui ne risquent pas d'être prisées par le seul grand public, avide d'être épaté par des charlatans de musiciens. Par contre, sans émettre de jugements explicites à leur égard, Platon semble, dans d'autres dialogues, se laisser amuser par les danses de divertissement, voire par les danses acrobatiques 57 . Ces danses rappellent, par leur caractère, les pratiques gymniques tellement honorées par le philosophe. Une autre catégorie de danses, également pratiquées pour les convives dans les banquets : de caractère bachique, elles étaient particulièrement aptes à aviver l'orgie du kômos. C'étaient là les danses conviviales que réprouvaient les sages et que les vases peints nous ont conservées en de multiples spécimens ${ }^{58}$, et susceptibles de dégénérer en manifestations de bas niveau. Leur caractère "politique", comme celui des danses pratiquées par les joueuses de flûte ${ }^{59}$, à supposer qu'il existât, serait déjà très atténué. Elles sont censées être des miméseis de "corps laids" et constituent une transition qualitative vers les danses orgiastiques proprement dites.

Ces dernières étaient accompagnées par le tympanon et surtout par l'aulos qui, en émettant des mélodies "de l'épouvante», causait la transe de la personne qui en était le destinataire. Dans l'Héraclès d'Euripide, Lyssa menace le héros de jouer sur l'aulos un tel air d'effroi à son adresse que, en le mettant hors de lui, il lui dictera des mouvements désordonnés, avant de le pousser à sa perte ${ }^{60}$. Il y a un rapport évident entre les danses orgiaques déclenchées par le cri exaspéré et terrifant de l'aulos et la peur causée chez le possédé. On peut expliquer ce phénomène en recourant à un mysticisme hypothétique, notamment à une conception de participation: "Liessence même de la possession, la docilité à répondre à cet appel démoniaque et à l'invitation de cette danse de l'au-delà ${ }^{61}$, voilà ce qu'on trouve sous l'apparente folie du possédé. Dans le cas de Platon, le caractère terrible de la musique orgiastique aurait vraisemblablement conduit à la conception de l'existence d'une âme défaillante chez le possédé. Il se peut aussi que ce

pragmatisme?, in La représentation. Actes du 17e Congrès de l'A.S.P.L.F. (1980), Strasbourg, 1981, p. 300-302.

56 Platon, Lois, III, 700b sq.

57 ID., Euthyd., 294e; Banquet, 190a.

58 L. SECHAN, op. cit., p. 227; 242 et la n. 38.

59 Platon, Banquet, $176 \mathrm{e}$.

60 Eur., Héraclès, 891-895.

61 H. Jeanmaire, Le Satyre et la Ménade..., art. cit., p. 473. 
même caractère, associé aux pratiques orgistiques, ait contribué à inspirer au philosophe son aversion pour l'aulos.

Dans l'ensemble, Platon semble classer les danses en deux catégories dont l'une comprend les danses décentes, l'autres les danses indécentes ${ }^{62}$. On serait tenté de l'admettre, mais tout en précisant qu'elles «relèvent d'un ordre de choses sur lequel le philosophe ne se sent pas une liberté complète d'expression ${ }^{63} \%$. Si elles sont jugées négativement, ce n'est plus parce qu'elles font simplement appel à des miméseis de "corps laids». "Il s'agit assurément de mimique, mais de mimique involontaire... Les danses de caractère orgiaque sont des danses de possédés ${ }^{64 . " ~ E l l e s ~ a p p a r t i e n n e n t ~ a ̀ ~ u n ~ g e n r e ~ n o n ~ p o l i t i q u e ~ q u i ~}$ échappe d'ailleurs à toute tentative de classification fixe. On ne saurait l'insérer nettement dans le système des danses que Platon s'est constitué. On constate même une volonté de sa part de garder le silence au sujet de tout un ensemble de données qui ont trait à un univers infernal et qui, de loin, exerce encore sur lui un attrait proche de la crainis et difficile à combattre ${ }^{65}$.

Non politique est une qualification qui, dans l'esprit de Platon, équivaut "avant la lettre» à la notion de "tabou». Ceci est également vrai de la musique envisagée au sens strict. Mais c'est à travers le traitement analytique que le philosophe fait subir à la notion de danse orgiastique qu'on trouve la clé appropriée pour décoder sa pensée relative aux états orgiastiques entendus dans l'acception la plus vaste. Aussi ces états sont-ils, pour ainsi dire, des états "privilégiés", parce qu'ils permettent aux fidèles de "participer» directement ${ }^{66}$ de la divinité à laquelle ils se sont voués et de vivre intensément sa présence. En outre, à un niveau méthodologique, parce qu'étant des états limites, ils permettent une meilleure approche et une explication plus poussée du mécanisme et $d u$ fonctionnement des homologies musicales. Rationaliste convaincu, Platon aurait peut-être aimé s'en tenir aux seuls rapports, de style apollinien, existant entre le fidèle et les divinités officiellement admises dans sa Cité. Il ne s'en sent toutefois pas

62 C. RitTer, Platos Gesetze, 1896, p. 210.

63 H. JeAnMaire, Le Satyre et la Ménade..., art. cit., p. 465.

64 Ibid., p. 466.

65 Eur., Hipp., 143, où il est question des "Corybantes respectables» et où "respectables» est censé rendre hommage à ces divinités. Sur les danses des mystères chez Platon, cf. H. Koller, Die Mimesis in der Antike. Nachahmung, Darstellung, Ausdruck, Bern, 1954, p. 42 sq. Sur les danses orgiastiques, ibid., p. 126 sq.

66 E. Moutsopoulos, Sur la notion de «participation musicale»..., art. cit. 
capable. S'il passe sous silence les rapports entre fidèles et divinités d'ordre bachique, voire infernal, c'est qu'il est empêché de rejeter celles-ci totalement, et préfere se taire : "Quant au reste, je me tais», déclarait Iphigénie ${ }^{67}$. Omniprésence du mystère? Encore une fois «avant la lettre», Platon tiendrait-il un «pari pascalien»?

Hypsilantou 40

E. MOUTSOPOULOS

GR - 11521 Athènes

67 Eur., Iph. Taur., 179-184. Cf. supra et la n. 2. 